\title{
On Tumbling of a Two-Dimensional Plate under Free Flight*
}

\author{
Katsuya HIRATA**, Masashi HAYAKAWA** and Jiro FUNAKI** \\ ** Department of Mechanical Engineering, Doshisha University, Kyoto 610-0321, Japan \\ E-mail: khirata@mail.doshisha.ac.jp
}

\begin{abstract}
In this study, we experimentally deal with the tumbling, which is a rotating motion of a plate with its axis perpendicular to the falling direction. As the plate, we consider a rectangular-cross-section prism with a depth-to-width ratio $\lambda=0.3$, an aspect ratio $A R=10$, and inertia moment ratios $I^{*}=0.75-43$, together with a wide range of a non-dimensional control parameter $C=7.2 \times 10^{1}-1.6 \times 10^{3}$. As a result, we specify fundamental aerodynamic characteristics in terminal condition, like the reduced rotating rate $\Omega^{*}$, the lift coefficient $C_{L}$, the drag coefficient $C_{D}$ and the lift-to-drag ratio $C_{L} / C_{D}$ as functions of both $C$ and $I^{*}$. Moreover, the relation between the actual Reynolds number $\operatorname{Re}$ and the Reynolds number $\operatorname{Re}\left(V_{d}\right)$ based on $V_{d}$ can be approximately determined, being independent of $C$ and $I^{*}$. Eventually, we successfully propose a series of empirical formulae to predict $\Omega^{*}, C_{L}, C_{D}$ and $C_{L} / C_{D}$ as functions of $\operatorname{Re}\left(V_{d}\right)$ alone, which is available for a wide parameter range except for large $C$ and small $I^{*}$.
\end{abstract}

Key words: Autorotation, Flat Plate, Free Flight, Free Fall, Tumbling

\section{Introduction}

When a solid matter falls in fluid, it's motion is usually complicated and sometimes experiences the autorotations, namely, various kinds of self-induced rotational motions sustained by fluid force (see Lugt $(1983)^{(1)}$ and Yoshinaga \& Tate $\left.(1985)^{(2)}\right)$. Among the autorotations such as the tumbling, the flat spin and the corning, we consider the tumbling, which is a rotational motion of a solid matter in fluid with the axis perpendicular to the falling direction. The tumbling is important not only for pure academic interests in leaves' and cards' falling motions, but also for aeronautics, space engineering, ballistics and meteorology.

So, there have existed some previous researches ${ }^{(3)}$ - (14) on the tumbling (for further details, see our previous work (2009) ${ }^{(15)}$ ). However, by these researches, we cannot know even fundamental relations between the important physical quantities representing aerodynamic characteristics and the governing parameters. Here, as the aerodynamic-characteristic quantities, we usually consider the reduced rotating rate $\Omega^{*}$, the lift coefficient $C_{L}$, the drag coefficient $C_{D}$, the lift-to-drag ratio $C_{L} / C_{D}$ and so on. - The relations reported in those previous researches are not accurate enough, because they are based on the experiments at small aspect ratio $A R=0.25-6.0$, and because all the governing parameters are not specified completely. - For example, though $\Omega^{*}$ is considerably effective upon the vicinal flow field and then upon the other aerodynamic characteristics, it is difficult to determine the precise value of $\Omega^{*}$ in enough terminal condition by both wind-tunnel experiments and computations. The difficulties by the former and the latter are owing to supporting systems, and owing to the strong non-linearity and 
three-dimensionality related with flow separations, respectively.

Recently, we have carried out a series of free-flight tests in air, and revealed the relations of $\Omega^{*}, C_{L}, C_{D}$ and $C_{L} / C_{D}$ in terminal condition with a pair of non-dimensional governing parameters $C$ and $I^{*}$, a pair of $C$ and $\operatorname{Re}(V)$, or a pair of $I^{*}$ and $\operatorname{Re}(V)$, where $C, I^{*}$ and $\operatorname{Re}(V)$ will be denoted later. However, in this pioneering work, the value of $C$ is kept to be constant to $3.9 \times 10^{2}$. Moreover, the work concerns relatively high Reynolds number $R e$, to reveal the whole features over wide ranges of parameters including $R e e^{(15)}$

So, in the present study, we methodically conduct many free-flight test in air and water, in order to determine $\Omega^{*}, C_{L}, C_{D}$ and $C_{L} / C_{D}$ in terminal condition, and attempt to reveal the relation of them with a non-dimensional governing parameter $C$, in addition to $I^{*}$ or $\operatorname{Re}\left(V_{d}\right)$. - About the definition of $\operatorname{Re}\left(V_{d}\right)$, see later- - In particular, we focus our attention upon a low-Re range, in order to specify more precise relations. Because, in such a low-Re range, the $R e$ effects upon the tumbling characteristics become larger. ${ }^{(15)}$

\section{Nomenclature}

$\begin{array}{lll}A R & : \text { Aspect ratio, } \equiv l / w & {[-]} \\ C & : \text { Non-dimensional control parameter, } \equiv\left(g w^{3}\right)^{1 / 2} / v & {[-]} \\ C_{D} & : \text { Drag coefficient, } \equiv D /\left(0.5 \rho_{\mathrm{f}} U_{\infty}{ }^{2} l w\right) & {[-]} \\ C_{L} & : \text { Lift coefficient, } \equiv L /\left(0.5 \rho_{\mathrm{f}} U_{\infty}{ }^{2} l w\right) & {[-]} \\ d & : \text { Plate depth } & {[\mathrm{m}]} \\ D & : \text { Drag } & {[\mathrm{N}]} \\ g & : \text { Gravity acceleration } & {\left[\mathrm{m} / \mathrm{s}^{2}\right]} \\ I & : \text { Inertia moment } & {\left[\mathrm{kg} \mathrm{m}{ }^{2}\right]} \\ I^{*} & : \text { Inertia moment ratio, } \equiv 32 I /\left(\rho_{\mathrm{f}} w^{4} l\right) & {[-]} \\ l & : \text { Plate span } & {[\mathrm{m}]} \\ L & : \text { Lift } & {[\mathrm{N}]} \\ n & : \text { Rotating rate } & {\left[\mathrm{s}^{-1}\right]} \\ \operatorname{Re} & : \text { Reynolds number, } \equiv U_{\infty} w / v & {[-]} \\ \operatorname{Re}(V), \operatorname{Re}\left(V_{d}\right) & : \text { Reynolds number based on } V, \text { or } V_{d} & {[-]} \\ t & : \text { Time } & {[\mathrm{s}]} \\ U_{\infty} & : \text { Mean translation velocity } & {[\mathrm{m} / \mathrm{s}]} \\ V, V_{d} & : \text { Theoretical velocity } & {[\mathrm{m} / \mathrm{s}]} \\ w & : \text { Plate width } & {[\mathrm{m}]} \\ x & : \text { Holizontal coordinate perpendicular to the rotation axis } & {[\mathrm{m}]} \\ y & : \text { Vertical coordinate } & {[\mathrm{m}]} \\ z & : \text { Horizontal coordinate parallel to the rotation axis } & {[\mathrm{m}]} \\ \lambda & : \text { Depth-to-width ratio, } \equiv d / w & {[-]} \\ v & : \text { Kinematic viscosity } & {\left[\mathrm{m}^{2} / \mathrm{s}\right]} \\ \rho_{\mathrm{f}} & : \text { Fluid density } & {\left[\mathrm{kg} / \mathrm{m}^{3}\right]} \\ \rho_{\mathrm{s}} & : \text { Plate density } & {\left[\mathrm{kg} / \mathrm{m}^{3}\right]} \\ \Omega^{*} & : \text { Reduced rotating rate, } \equiv \pi n w / U_{\infty} & {[-]} \\ \Omega^{*}{ }_{\mathrm{AOR}} & : \Omega^{*} \text { averaged over one plate's rotation } & {[-]} \\ & & \end{array}$

\section{Experimental Method}

\subsection{Model}

Figure 1 shows a test plate; namely, a prism with a rectangular cross section. Here, $l, w$ and $d$ denote span, width and depth of the plate, respectively. Figure 2 shows a schematic diagram of the tumbling motion of the plate and acting forces, together with the present coordinate system. 
The acting forces $W, L$ and $D$ are the weight, lift and drag on the plate, respectively. The resultant force $R$ of both $L$ and $D$ is the same as $-W$ in the terminal condition. Mean translation velocity $U_{\infty}$ is parallel to the mean falling direction of the plate. We specify $W$ from the model mass considering the buoyancy force, and both $L$ and $D$ from the glide angle and $W$.

$x$ and $z$ represent horizontal coordinates perpendicular and parallel to the plate's rotation axis, respectively. And, $y$ represents a vertical coordinate.

\subsection{Governing parameters}

Governing parameters are classified into two; namely, geometric and kinematic ones. At first, we consider the former. Referring to Fig. 1, we choose the depth-to-width ratio $\lambda$ and the aspect ratio $A R$ as the geometric governing parameters. The definitions of $\lambda$ and $A R$ are given by the following equations.

$$
\begin{aligned}
& \lambda \equiv \frac{d}{w} . \\
& A R \equiv \frac{l}{w} .
\end{aligned}
$$

In all the present experiments in both air and water, $\lambda$ and $A R$ are fixed to 0.3 and 10 , respectively.

Complementally speaking, we have to consider the condition of the plate's ends, because it influences the three-dimensionality of flow. In the present study, we do not put any end plates which ensure the two-dimensionality of flow. Instead, we keep an enough large value of $A R(=10)$ through all the tests, to ignore the $A R$ effects. - About the $A R$ effects, see Hirata et al. (2009). ${ }^{(15)}$

Second, we consider the kinematic governing parameters. We need two non-dimensional parameters to identify the concerning phenomenon. Conventionally, we select the inertia moment ratio $I^{*}$ and the Reynolds number $R e$ (for example, see Smith $\left.(1971)^{(6)}\right)$. The definitions of $I^{*}$ and $R e$ are as follows:

$$
I^{*} \equiv \frac{32 I}{\pi \rho_{\mathrm{f}} w^{4} l},
$$

and

$$
R e \equiv \frac{U_{\infty} w}{v},
$$

where $I$ denotes the inertia moment of the plate given by

$$
I \equiv \frac{\rho_{\mathrm{s}} w d l\left(w^{2}+d^{2}\right)}{12} .
$$

Here, $\rho_{\mathrm{f}}, \rho_{\mathrm{s}}$ and $v$ denote fluid density, plate density and kinematic viscosity, respectively.

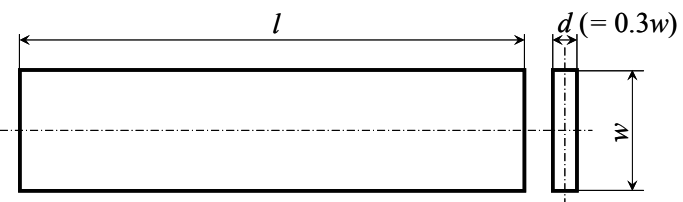

Fig. 1 Tumbling plate; a prism with a rectangular cross section.

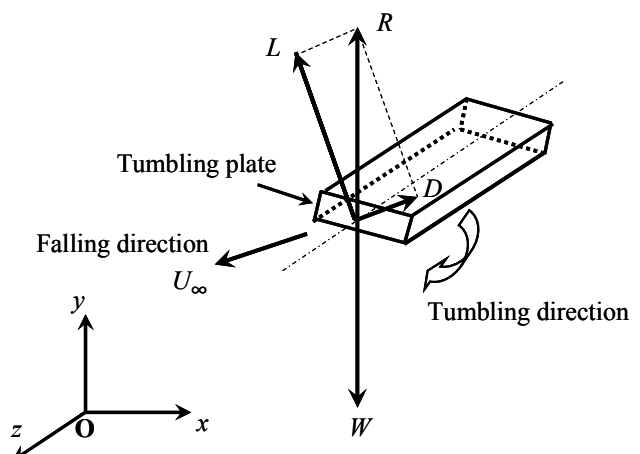

Fig. 2 Schematic diagram of the tumbling of a plate and acting forces, together with the coordinate system. 
From a theoretical point of view, we have to independently investigate each effect of such two governing parameters as $I^{*}$ and Re. However, in actual free-flight experiments, individual estimations to such kinematic governing parameters are technically difficult. This has been just one main reason to prevent the understanding of the concerning phenomenon. -For example, in Iversen (1979), each parameter effect does not estimated separately from the other parameter effect, under the condition that the other parameter is not kept to be constant.-

In the present study, as well as Hirata et al. (2009), for the individual estimations to kinematic governing parameters avoiding such a difficulty, we choose the following non-dimensional control parameter $C$, instead of $R e$.

$$
C \equiv \frac{\sqrt{g w^{3}}}{v} \text {. }
$$

From a dimensional analysis, $C$ represents the ratio of (inertia force $\times$ gravity force $)^{1 / 2}$ to viscous force. And, from a practical point of view, $C$ can be easily controlled in experiments. Then, in the present study, we fix $C$ to one among six constant values ranging from $7.2 \times 10^{1}$ to $1.6 \times 10^{3}$, and investigate the pure effects of both $C$ and the other kinematic governing parameter $I^{*}$.

We may consider another Reynolds number $\operatorname{Re}(V)$ or $\operatorname{Re}\left(V_{d}\right)$, instead of $C, I^{*}$ or $\operatorname{Re}$. $\operatorname{Re}(V)$ and $\operatorname{Re}\left(V_{d}\right)$ are the Reynolds numbers based on theoretical velocity $V$ and $V_{d}$ instead of $U_{\infty}$, respectively. In the present study, we consider not $\operatorname{Re}(V)$, but $\operatorname{Re}\left(V_{d}\right)$ as well as Andersen et al. (2005) $)^{(13)}$. - $\operatorname{Re}\left(V_{d}\right)$ has an advantage in such a direct interpretation of $V_{d}$ as supposing $C_{D}=1$, while $\operatorname{Re}(V)$ has an advantage in simplicity of the formulation where we throughout use a sole characteristic length scale $w$. The relation of both is given by $\operatorname{Re}\left(V_{d}\right)=\sqrt{\lambda} \operatorname{Re}(V)$. - The definition of $\operatorname{Re}\left(V_{d}\right)$ is given by

$$
\operatorname{Re}\left(V_{d}\right) \equiv \frac{V_{d} w}{v} .
$$

And, the theoretical terminal velocity $V_{d}$ is as follows:

$$
V_{d} \equiv \sqrt{2\left(\frac{\rho_{\mathrm{s}}}{\rho_{\mathrm{f}}}-1\right) d g},
$$

where $g$ denotes the gravity acceleration.

In many practical aspects, we can easily find advantages in the use of $V_{d}$, instead of the use of $U_{\infty}$; namely, the use of $\operatorname{Re}\left(V_{d}\right)$ instead of the use of $\operatorname{Re}$. For example, we can calculate the value of $V_{d}$, once physical properties and geometrical dimensions are given. On the other hand, we need experiments or numerical simulations to determine $U_{\infty}$. From another viewpoint, the use of $V_{d}$ inevitably causes some lost information. As shown later, it will be experimentally confirmed that the uniqueness in the relation between $V_{d}$ and $U_{\infty}$ are approximately satisfied. Then, $V_{d}$ could be sufficient to understand the concerning phenomenon in perspective, and more convenient than $U_{\infty}$. We will also discuss the lost information, later.

Hereafter, as two kinematic governing parameters, we consider a pair of $C$ and $I^{*}$, a pair of $C$ and $\operatorname{Re}\left(V_{d}\right)$ or a pair of $I^{*}$ and $\operatorname{Re}\left(V_{d}\right)$, instead of a pair of $I^{*}$ and $\operatorname{Re}$. In the following section, supposing technical difficulties in actual experiments, we will first attempt a pair of $C$ and $I^{*}$. Second, we will attempt a pair of $C$ and $\operatorname{Re}\left(V_{d}\right)$ or a pair of $I^{*}$ and $\operatorname{Re}\left(V_{d}\right)$.

Incidentally, the relation among $C, I^{*}$ and $\operatorname{Re}\left(V_{d}\right)$ is given by

$$
\frac{\operatorname{Re}\left(V_{d}\right)}{C}=\sqrt{2 \lambda\left(\frac{3 \pi}{8} \cdot \frac{I^{*}}{\lambda+\lambda^{3}}-1\right)},
$$

where $\lambda=0.3$.

In Table 1, we summarise the values of governing parameters by the authors and other researchers, together with the corresponding symbols used in figures. And, Table 2 shows 
Table 1 Parameters and the corresponding symbols in Figs. $5-15$.

\begin{tabular}{c|c|c|c|c|c|c}
\hline Reference & Symbol & $\lambda$ & $A R$ & $C$ & $I^{*}$ & Experimental method \\
\hline Present & $\bigcirc, \square, \Delta, \diamond$ & 0.3 & 10 & $7.2 \times 10^{1}$ & $4.2-42$ & free flight in air \\
\hline Present & $\bigcirc, \square, \triangle, \diamond$ & 0.3 & 10 & $1.1 \times 10^{2}$ & $4.7-43$ & free flight in air \\
\hline Present & $\bigcirc, \square, \triangle, \diamond$ & 0.3 & 10 & $2.0 \times 10^{2}$ & $4.2-36$ & free flight in air \\
\hline Present & $\bigcirc, \square, \triangle, \diamond$ & 0.3 & 10 & $3.7 \times 10^{2}$ & $3.5-43$ & free flight in air \\
\hline Present & $\bigcirc, \square$ & 0.3 & 10 & $4.7 \times 10^{2}$ & $0.75-2.5$ & free flight in water \\
\hline Present & $\bigcirc, \square$ & 0.3 & 10 & $\begin{array}{c}1.3 \times 10^{3}- \\
1.6 \times 10^{3}\end{array}$ & $0.75-2.5$ & free flight in water \\
\hline $\begin{array}{c}\text { Hirata et al. } \\
(2009)\end{array}$ & $\times$ & 0.3 & $2.0-20$ & $3.9 \times 10^{2}$ & $3.3-141$ & free flight in air \\
\hline $\begin{array}{c}\text { Dupleich (1949) } \\
\text { quoted by } \\
\text { Poreh \& Wray } \\
\text { (1979) }\end{array}$ & + & $\begin{array}{c}\text { unknown } \\
(0.003-0.01)\end{array}$ & $1.0-5.0$ & $\begin{array}{c}1.4 \times 10^{3}- \\
6.5 \times 10^{3}\end{array}$ & $1.60-4.50$ & $\begin{array}{c}\text { free flight in air } \\
\text { Bustamante \& } \\
\text { Stone (1969) }\end{array}$ \\
\hline
\end{tabular}

Table 2 Details of $C, I^{*}$ and $\operatorname{Re}\left(V_{d}\right)$, together with corresponding symbols in Figs. 5 - 15. In all the present experiments, $\lambda=0.3$ and $A R=10$.

\begin{tabular}{c|c|c|c|c|c}
\hline Reference & Symbol & $C$ & $I^{*}$ & $R e\left(V_{d}\right)$ & $R e$ \\
\hline Present & $\bigcirc$ & $7.2 \times 10^{1}$ & 4.2 & 220 & $1.9 \times 10^{2}-2.2 \times 10^{2}$ \\
\hline Present & $\square$ & $7.2 \times 10^{1}$ & 6.1 & 250 & $2.2 \times 10^{2}-2.5 \times 10^{2}$ \\
\hline Present & $\triangle$ & $7.2 \times 10^{1}$ & 10 & 320 & $2.4 \times 10^{2}-2.7 \times 10^{2}$ \\
\hline Present & $\diamond$ & $7.2 \times 10^{1}$ & 42 & 700 & $5.3 \times 10^{2}-5.8 \times 10^{2}$ \\
\hline Present & $\bigcirc$ & $1.1 \times 10^{2}$ & 4.7 & 350 & $2.7 \times 10^{2}-2.9 \times 10^{2}$ \\
\hline Present & $\square$ & $1.1 \times 10^{2}$ & 7.3 & 450 & $3.3 \times 10^{2}-3.4 \times 10^{2}$ \\
\hline Present & $\triangle$ & $1.1 \times 10^{2}$ & 13 & 530 & $4.0 \times 10^{2}-4.8 \times 10^{2}$ \\
\hline Present & $\diamond$ & $1.1 \times 10^{2}$ & 43 & 1090 & $8.8 \times 10^{2}-9.2 \times 10^{2}$ \\
\hline Present & $\bigcirc$ & $2.0 \times 10^{2}$ & 4.2 & 600 & $4.3 \times 10^{2}-4.5 \times 10^{2}$ \\
\hline Present & $\square$ & $2.0 \times 10^{2}$ & 7.4 & 730 & $5.7 \times 10^{2}-5.9 \times 10^{2}$ \\
\hline Present & $\triangle$ & $2.0 \times 10^{2}$ & 12 & 970 & $6.8 \times 10^{2}-7.2 \times 10^{2}$ \\
\hline Present & $\diamond$ & $2.0 \times 10^{2}$ & 36 & 1830 & $1.2 \times 10^{3}-1.3 \times 10^{3}$ \\
\hline Present & $\bigcirc$ & $3.7 \times 10^{2}$ & 3.5 & 1000 & $7.0 \times 10^{2}-7.8 \times 10^{2}$ \\
\hline Present & $\square$ & $3.7 \times 10^{2}$ & 6.3 & 1300 & $9.9 \times 10^{2}-1.0 \times 10^{3}$ \\
\hline Present & $\triangle$ & $3.7 \times 10^{2}$ & 22 & 2530 & $1.8 \times 10^{3}-1.9 \times 10^{3}$ \\
\hline Present & $\diamond$ & $3.7 \times 10^{2}$ & 43 & 3560 & $2.6 \times 10^{3}-2.9 \times 10^{3}$ \\
\hline Present & $\bigcirc$ & $4.7 \times 10^{2}$ & 0.75 & 480 & $3.3 \times 10^{2}-3.4 \times 10^{2}$ \\
\hline Present & $\square$ & $4.7 \times 10^{2}$ & 2.5 & 1040 & $7.3 \times 10^{2}-7.4 \times 10^{2}$ \\
\hline Present & $\bullet$ & $1.6 \times 10^{3}$ & 0.75 & 1650 & $1.1 \times 10^{3}-1.3 \times 10^{3}$ \\
\hline Present & $\square$ & $1.3 \times 10^{3}$ & 2.5 & 3030 & $2.2 \times 10^{3}-2.3 \times 10^{3}$ \\
\hline Hirata et al. & $\times$ & $3.9 \times 10^{2}$ & $3.3-141$ & $980-12300$ & $1.8 \times 10^{3}-6.4 \times 10^{3}$ \\
(2009) & $\times$ & & & & \\
\hline & & & & & \\
\hline
\end{tabular}

the details of the present kinematic governing parameters, together with the corresponding symbols.

\subsection{Aerodynamic Characteristics}

As dependent variables of the above governing parameters, we consider physical quantities describing the tumbling characteristics in terminal condition, such as a rotating rate $n$ and the lift $L$ and the $\operatorname{drag} D$ of the plate. They are non-dimensionalised as a reduced rotating rate $\Omega^{*}$, a lift coefficient $C_{L}$ and a drag coefficient $C_{D}$, respectively. Strictly speaking, they are all the ensemble-means of the time-averaged values over several rotations in terminal condition; except for $\Omega^{*}{ }_{\text {AOR }}$ which will be shown only in Fig. 3. Their 
definitions are as follows.

$$
\begin{aligned}
\Omega^{*} & \equiv \frac{\pi n w}{U_{\infty}} . \\
C_{L} & \equiv \frac{L}{\frac{1}{2} \rho_{\mathrm{f}} U_{\infty}{ }^{2} w l} . \\
C_{D} & \equiv \frac{D}{\frac{1}{2} \rho_{\mathrm{f}} U_{\infty}{ }^{2} w l} .
\end{aligned}
$$

From Eq. (10), we see that $\Omega^{*}$ represents the ratio of the plate's tip velocity to the plate's translation velocity $U_{\infty}$ in terminal condition. Besides, we consider another important aerodynamic-characteristics quantity $C_{L} / C_{D}$ which is referred to as the lift-to-drag ratio, in addition to $\Omega^{*}, C_{L}$ and $C_{D}$.

Hereafter, we investigate physical quantities like $\Omega^{*}, C_{L}, C_{D}$ and $C_{L} / C_{D}$ as functions of a pair of $C$ and $I^{*}$, a pair of $C$ and $\operatorname{Re}\left(V_{d}\right)$ or a pair of $I^{*}$ and $\operatorname{Re}\left(V_{d}\right)$.

\subsection{Experimental apparatus}

The three-dimensional motion of a plate is detected by a synchronised set of two or three cameras, whose foci are fixed near the prospected orbit of the plate. We use one of the cameras only to judge the exact two-dimensionality of the motion. The camera is placed on the prospected-orbit plane. By this operation, we can ensure the two-dimensionality on a higher level than the previous work ${ }^{(15)}$.

In order to record the motion just in terminal condition, we choose the space enough below the launched position as a test space. On the other hand, the test space is enough above the bottom floor to avoid the ground effect. Of course, we check whether or not the recorded motion is terminal, prior to their analyses.

Figures 3 and 4 show the schematics of experimental apparatus in air and in water, respectively.

In Fig. 3, a launched flat plate begins to fall tumbling. We take photographs by two high-speed video cameras synchronised with each other at 500 flames/s, and get the angle and the position of the plate.

In Fig. 4, a flat plate falls in a water tank with a water level of $7.0 \times 10^{-1} \mathrm{~m}$. The water tank has such dimensions as $9.7 \times 10^{-1} \mathrm{~m}$ in span, $1.3 \mathrm{~m}$ in width and $7.5 \times 10^{-1} \mathrm{~m}$ in height. The maximum blockage ratio $B R$ through all the experiments varies from $0.016 \%$ to $0.065 \%$, where $B R$ is defined by the maximum projected area of the model divided by the minimum vertical-cross-section area of water. The interval between experiments is kept to

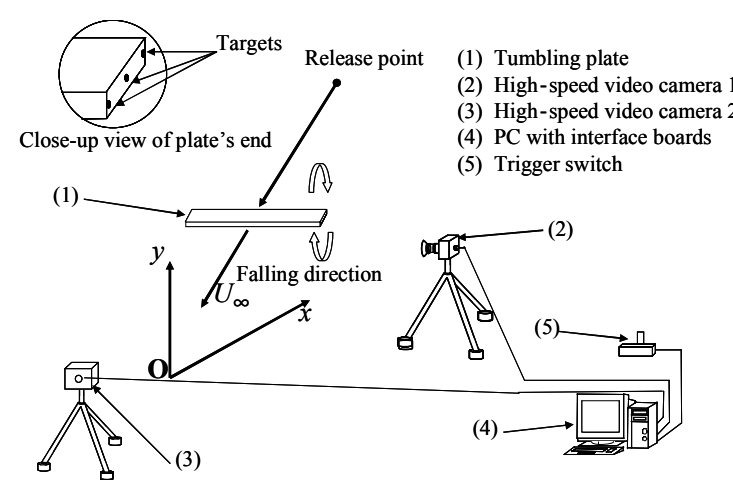

Fig. 3 Experimental apparatus in air.

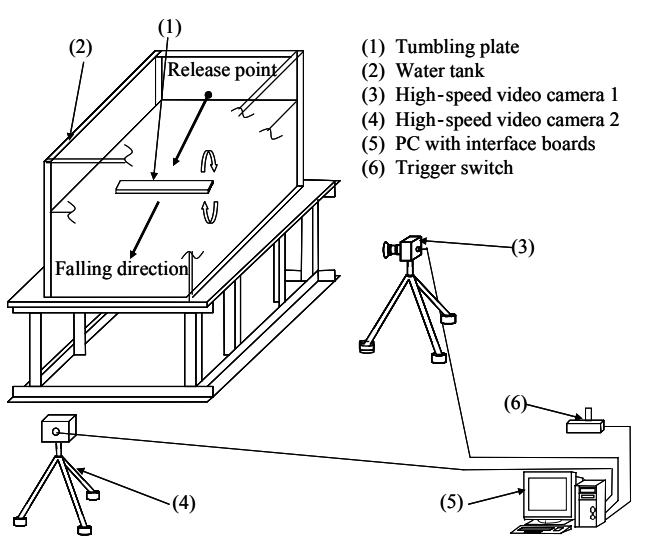

Fig. 4 Experimental apparatus in water. 
be enough long to settle the disturbances in the water tank. We take synchronised photographs by two or three high-speed video cameras at 500 frames/s, and get the angle and the position of the plate as well.

Specifically speaking, as targets, we mark three white dots on a longer cross-section centre line on one plate's end surface. One dot is on the centre, and the other two dots are oppositely outside the centre. The former is for the position measurements, and the latter two are for the angle measurements.

The plate has such dimensions as $l=5.0 \times 10^{-2}-1.5 \times 10^{-1} \mathrm{~m}, w=5.0 \times 10^{-3}-1.5 \times 10^{-2} \mathrm{~m}$ and $d=1.5 \times 10^{-3}-4.5 \times 10^{-3} \mathrm{~m}$ in the air experiments, or as $l=3.3 \times 10^{-2}-6.6 \times 10^{-2} \mathrm{~m}, \mathrm{w}=$ $3.3 \times 10^{-3}-6.6 \times 10^{-3} \mathrm{~m}$ and $d=1.0 \times 10^{-3}-2.0 \times 10^{-3} \mathrm{~m}$ in the water experiments.

The plates are made of foamed polystyrene, balsa wood, aluminum or cupper. We can control the plate's inertia moment $I$ by choosing such materials. - For the other details in experimental apparatus, see ref. ${ }^{(15)}$ _

\section{Results and Discussion}

\section{1 $\Omega_{\text {AOR }}^{*}$}

Among the four aerodynamic-characteristics quantities, $\Omega^{*}$ is supposed to be primarily influential to the tumbling. So, we first consider $\Omega^{*}$. Figure 5 shows $\Omega^{*}$ AOR plotted against $R e$, for several combinations of $C$ and $I^{*}$ whose values are described in Table 2, together with a low-Re part of the data in Hirata et al. (2009). ${ }^{(15)}$ Here, $\Omega^{*}$ AOR denotes the reduced rotating rate averaged not over enough long time but over only one plate's rotation.

From a qualitative viewpoint, the present results are similar with Hirata et al. That is, as $R e$ increases from zero, $\Omega_{\text {AOR }}^{*}$ monotonously increases approaching a constant value in a range of $0.4-0.6$. Of course, the range is the same as Hirata et al. where the range is independent of $R e$ at high $R e \gtrsim 4 \times 10^{3}$ as shown in Fig.5. Such a tendency can be also confirmed by a thick solid line described by a formula, which will be discussed later. Of course, such a tendency is also the same as Hirata et al. whose experimental data are denoted by cross symbols.

When we carefully see the data with the same $C$ and the same $I^{*}$, we notice that the data collapse near the thick solid line, not with a random scattering manner, but with a coherent scattering manner on a certain curve which has such a trend as $\Omega^{*}{ }_{\text {AOR }}$ decreases with increasing $R e$.

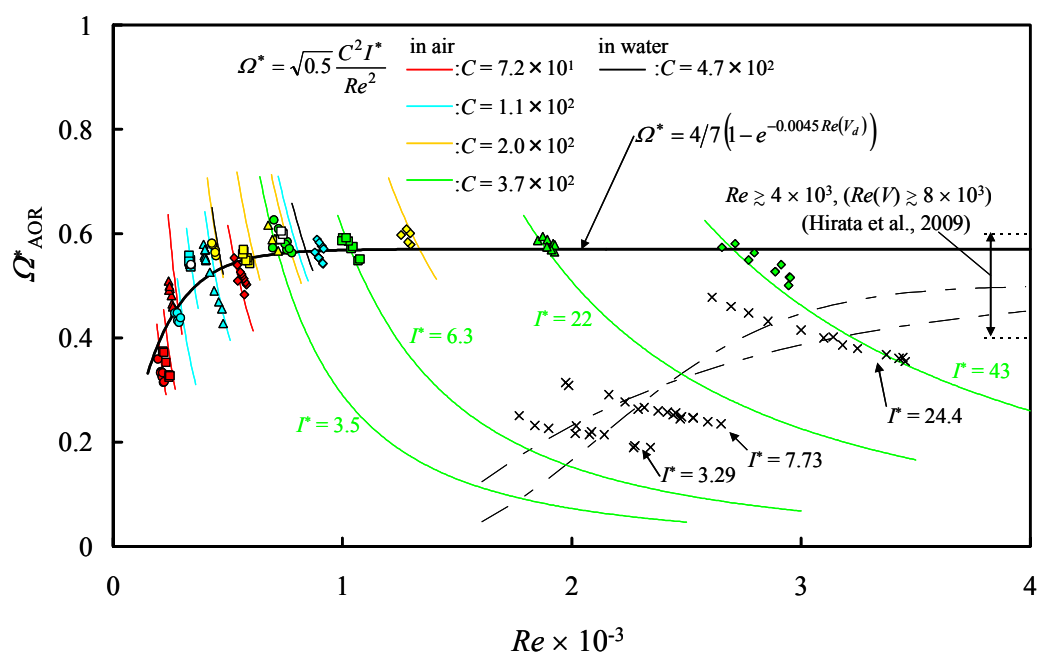

Fig. 5 Reduced rotating rate $\Omega_{\text {AOR }}^{*}$ averaged over one rotation versus Reynolds number $R e$, for $\lambda=0.3$, $A R=10, C=7.2 \times 10^{1}-4.7 \times 10^{2}$ and $I^{*}=0.75-43$. 
To conclude, it is considered that the scattering manner is reflected by a close interaction between $C_{L}$ and $\Omega^{*}{ }_{\text {AOR }}$. In Hirata et al., it has been found out that the scattering manner is well approximated by a semi-theoretical formula, which is based on the dynamical equilibrium on a free-flight plate supposing that the lift force $L$ is produced mainly by the Magnus effect. However, the formula is merely for a single value of $C=$ $3.9 \times 10^{2}$. So, we expand it, considering the effect of $C$ in addition to that of $I^{*}$. Now, we consider the dynamical equilibrium on a free-flight plate, and we get

$$
L^{2}+D^{2}=\left(\rho_{\mathrm{s}} l d w g\right)^{2}
$$

where $\rho_{\mathrm{s}}-\rho_{\mathrm{f}}$ is approximated by $\rho_{\mathrm{s}}$. We suppose that the lift force $L$ is produced mainly by the Magnus effect, then

$$
L \propto \rho_{\mathrm{f}} U_{\infty} n w^{2} l .
$$

And,

$$
D \propto \frac{1}{2} \rho_{\mathrm{f}} U_{\infty}^{2} w l .
$$

From Eqs. (13) - (15), we get

$$
\frac{n^{2} w^{2}}{U_{\infty}^{2}}+\frac{1}{4} \frac{C_{2}^{2}}{C_{1}^{2}}=\frac{1}{C_{1}^{2}} \frac{g^{2} d^{2}\left(\rho_{\mathrm{s}} / \rho_{\mathrm{f}}\right)^{2}}{U_{\infty}^{4}},
$$

where $C_{1}$ and $C_{2}$ are the coefficients which are related with Eqs. (14) and (15), respectively. From Eq. (16) together with Eqs. (1), (3) - (6) and (10), we get such a non-dimensionalised equation as

$$
\left(1+\frac{\pi^{2}}{4}\left(\frac{C_{2}}{C_{1}}\right)^{2} \frac{1}{\Omega^{*^{2}}}\right) \Omega^{*^{2}}=\frac{9 \pi^{4}}{64 C_{1}^{2}} \frac{1}{\left(1+\lambda^{2}\right)^{2}} \frac{C^{4} I^{*^{2}}}{R e^{4}} .
$$

If we suppose $C_{1}=\pi^{2}$ and $C_{2}=1$, then $\pi^{2} / 4\left(C_{2} / C_{1}\right)^{2}=0.025$. On the other hand, we can conform by experiments that $\Omega^{*}$ usually varies from 0.3 to 0.6 . Therefore, $1+\pi^{2} / 4\left(C_{1} / C_{2}\right)^{2} 1 / \Omega^{* 2}$ has a variable range of $19 \%$, while $\Omega^{* 2}$ has a variable range of $400 \%$. So, we simply approximate the left hand of Eq. (17) to $\Omega^{* 2}$, and get

$$
\Omega^{*}=A \frac{C^{2} I^{*}}{R e^{2}}
$$

with a coefficient

$$
A=\frac{3 \pi^{2}}{8} \frac{1}{C_{1}\left(1+\lambda^{2}\right)} .
$$

We experimentally find $\sqrt{0.5}$ as $A$. Of course, Eq. (18) with $C=3.9 \times 10^{2}$ well agrees with Hirata et al. In Fig. 5, we can confirm that thin solid lines representing Eq. (18) are in good agreements with the present data, over a wide range of $C=7.2 \times 10^{1}-4.7 \times 10^{2}$.

From a quantitative viewpoint, we see a discrepancy in the value of $\Omega_{\text {AOR }}^{*}$ between the present experiments and Hirata et al. The main reason is considered to be owing to less accuracy in the low-Re-range experiments in Hirata et al., where the plate's rotation axis could not be perfectly horizontal with the trajectory on an imperfect plane, because a wider $R e$ range including much higher $R e$ 's is their original concern.

\subsection{Influence of $C$ and $I^{*}$}

Now, we have confirmed the validity of a formula Eq. (18). However, we have to know the value of $R e$ in advance, if we use Eq. (18). In other words, only by Eq. (18), we can predict not the mean value $\Omega^{*}$ of $\Omega^{*}$ AOR but merely the scattering manner of $\Omega^{*}$ AOR on the $R e-\Omega^{*}$ plane. - As will be discussed in Fig. 15, Eq. (18) with the empirically-determined value of $R e$ gives us less accurate $\Omega^{*}$. - Then, in this subsection, we investigate both the $C$ effects and the $I^{*}$ effects upon $\Omega^{*}, C_{L}, C_{D}$ and $C_{L} / C_{D}$ in wide parameter ranges of $C$ and $I^{*}$. And, we attempt to find out an empirical formula to precisely predict the value of $\Omega^{*}$ together with those for $C_{L}, C_{D}$ and $C_{L} / C_{D}$, considering $C$ and $I^{*}$ as two kinematic governing 
parameters.

Figures 6, 7, 8 and 9 show the $I^{*}$ effects upon $\Omega^{*}, C_{L}, C_{D}$ and $C_{L} / C_{D}$ for several values of $C$, respectively, in the ranges of $C=7.2 \times 10^{1}-1.6 \times 10^{3}$ and $I^{*}=0.75-43$. And, Table 2 summarises the symbols and their corresponding parameters used in these figures.

In Fig. 6, we first see the data for $C=7.2 \times 10^{1} . \Omega^{*}$ monotonously increases with increasing $I^{*}$, asymptoting to a constant value between 0.4 and 0.6 , while there exist small data scatterings. We can observe such similar effects of $I^{*}$ upon $\Omega^{*}$ for the other five values of $C$ as that for $C=7.2 \times 10^{1}$. Strictly Speaking, $\Omega^{*}$ for $C=2.0 \times 10^{2}$ and $3.7 \times 10^{2}$ tends to have a constant value being independent of $I^{*}$, and does not increase with increasing $I^{*}$. Because, there are no data at enough small $I^{*}$ due to the technical difficulty to find out appropriate materials for the model. However, if we consider the results for $C=7.2 \times 10^{1}$ and $1.1 \times 10^{2}$, we might suppose the similar effects of $I^{*}$ upon $\Omega^{*}$.

Referring to all the data with six values of $C$, we propose an empirical formula given by

$$
\Omega^{*}=\alpha_{\Omega}\left(1-\beta_{\Omega} e^{-\gamma_{\Omega} I^{*}}\right),
$$

with $\alpha_{\Omega}=4 / 7, \beta_{\Omega}=0.27 C^{0.32}$ and $\gamma_{\Omega}=0.0006 C^{1.4}$. Thin solid lines in Fig. 6 represent this empirical formula Eq. (20). We can confirm that the lines are in good agreement with the experimental data. - More specifically, the agreement for $C=7.2 \times 10^{1}$ seems inferior to those for other $C$ 's. For this reason, we can suggest that the measurements for $C=7.2 \times 10^{1}$ are conducted at the smallest values of $R e$, where the tumbling tends to become unstable due to viscous effects. -

In Fig. 7, we first see the data for $C=7.2 \times 10^{1} . C_{L}$ monotonously increases with increasing $I^{*}$, asymptoting to a constant value of about $7 / 5$, while there exist some data scatterings. We can observe such similar effects of $I^{*}$ upon $C_{L}$ for $C=1.1 \times 10^{2}$ and $2.0 \times 10^{2}$ as that for $C=7.2 \times 10^{1}$. However, for $C \geq 3.7 \times 10^{2}, C_{L}$ monotonously decreases with increasing $I^{*}$, asymptoting to about $7 / 5$. Referring to all the data with six values of $C$, we propose an empirical formula given by

$$
C_{L}=\alpha_{L}\left(1-\beta_{L} e^{-\gamma_{L} I^{*}}\right)
$$

with $\alpha_{L}=7 / 5, \beta_{L}=1800 C^{-1.4}-0.5$ and $\gamma_{L}=0.5$. Thin solid lines in Fig. 7 represent this empirical formula Eq. (21). We can confirm that the lines are in good agreement with the experimental data, as well. - More specifically, the agreement for $C=7.2 \times 10^{1}$ seems inferior to those for other $C$ 's, as well as Fig. 6.-

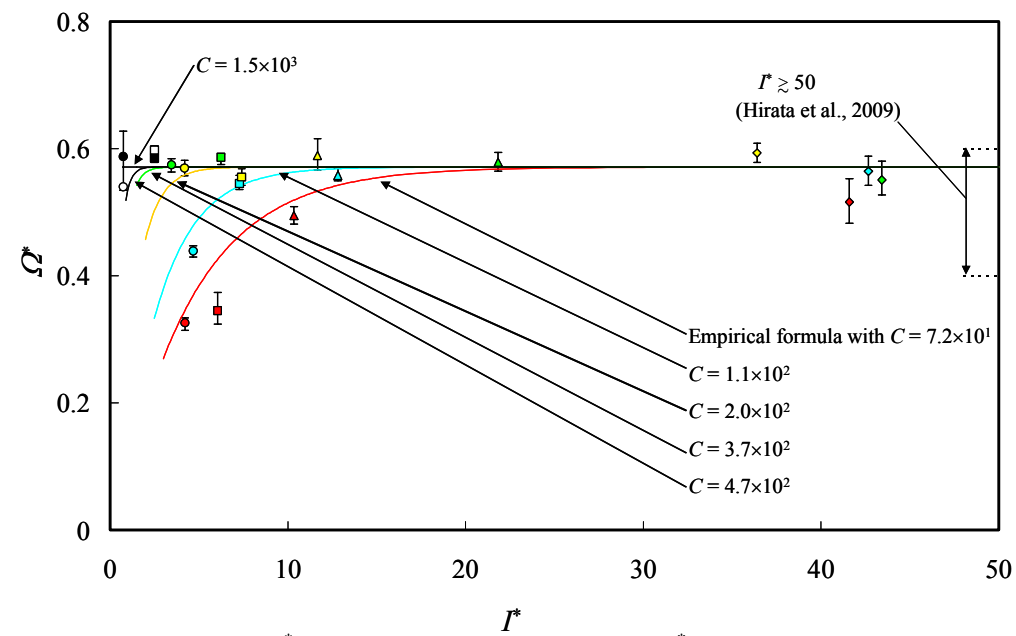

Fig. 6 Reduced rotating rate $\Omega^{*}$ of versus inertia moment ratio $I^{*}$, for $\lambda=0.3, A R=10, C=7.2 \times 10^{1}-$ $1.6 \times 10^{3}$ and $I^{*}=0.75-43$. Empirical formula is described as Eq. (20). 


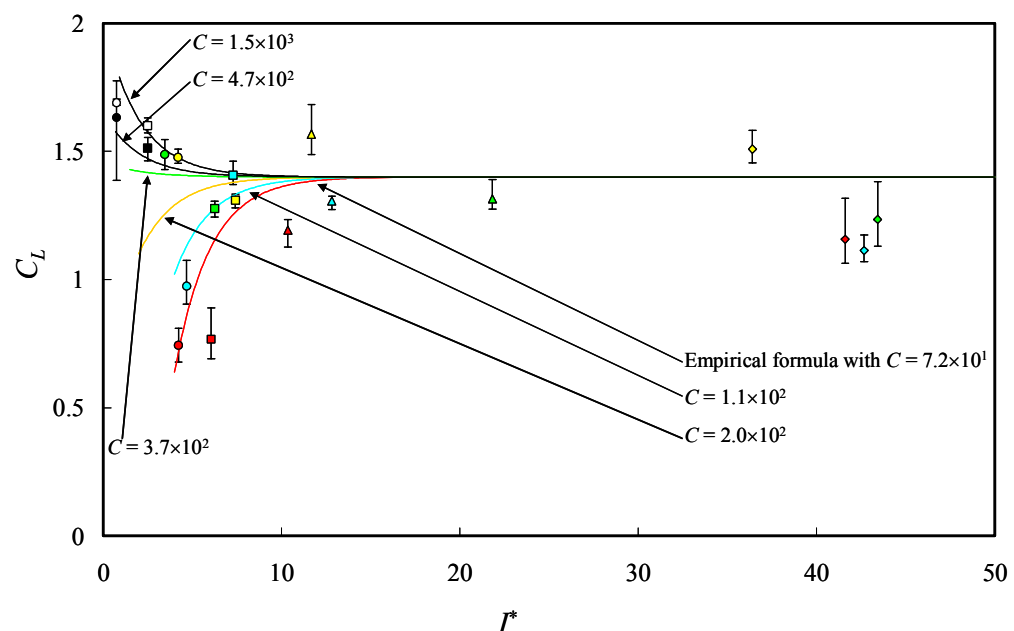

Fig. 7 Lift coefficient $C_{L}$ versus inertia moment ratio $I^{*}$, for $\lambda=0.3, A R=10, C=7.2 \times 10^{1}-1.6 \times 10^{3}$ and $I^{*}$ $=0.75-43$. Empirical formula is described as Eq. (21).

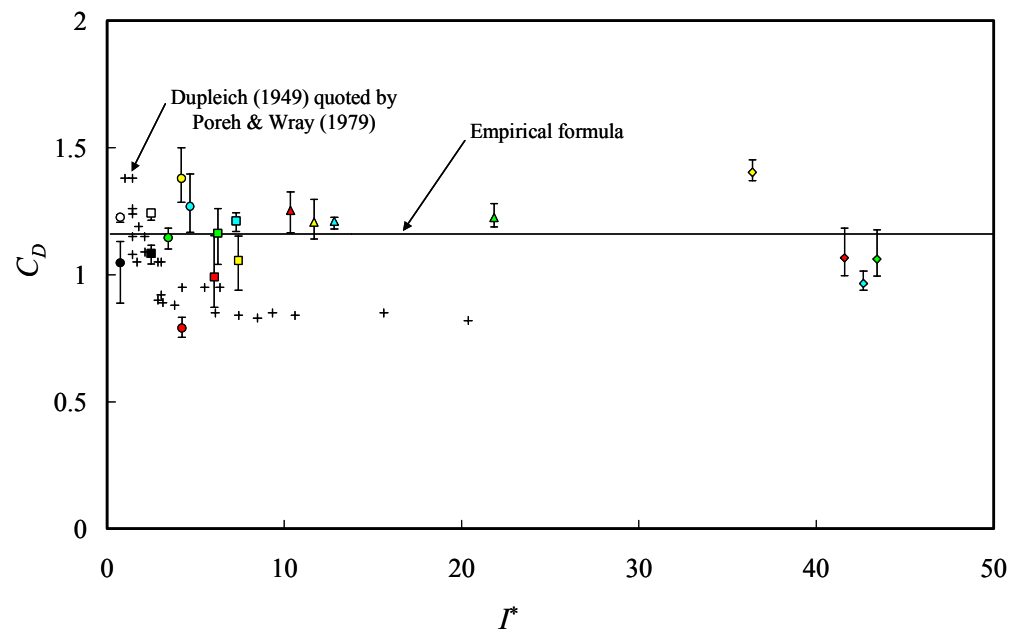

Fig. 8 Drug coefficient $C_{D}$ versus inertia moment ratio $I^{*}$, for $\lambda=0.3, A R=10, C=7.2 \times 10^{1}-1.6 \times 10^{3}$ and $I^{*}=0.75-43$. Empirical formula is described as Eq. (22).

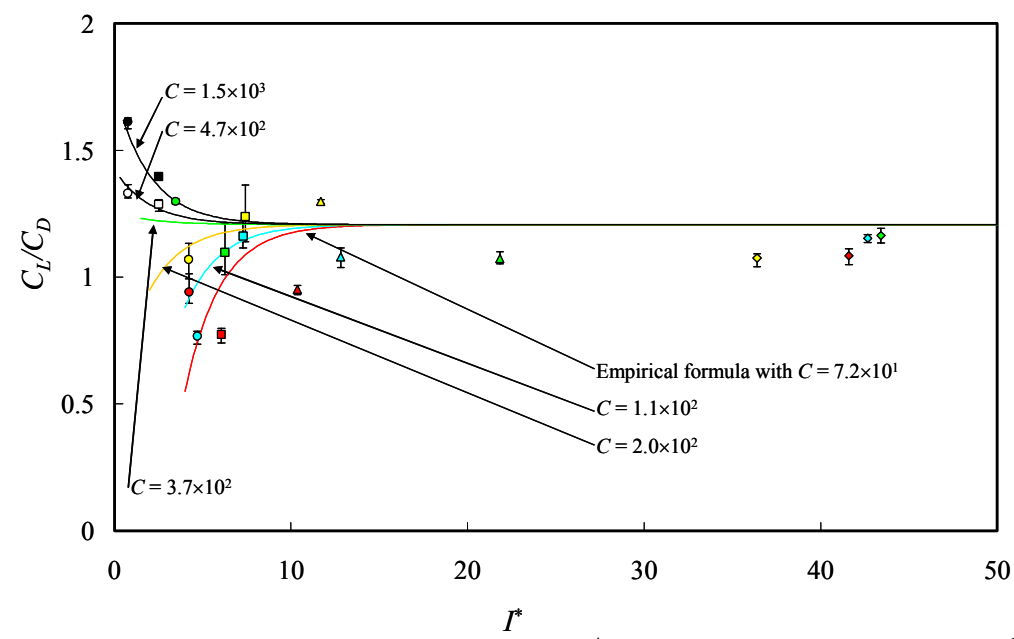

Fig. 9 Lift-drag ratio $C_{L} / C_{D}$ versus inertia moment ratio $I^{*}$, for $\lambda=0.3, A R=10, C=7.2 \times 10^{1}-1.6 \times 10^{3}$ and $I^{*}=0.75-43$. Empirical formula is described as Eq. (23). 
In Fig. $8, C_{D}$ tends to scatter is a range centering about $7 / 6$, while there exist some data scatterings, being independent of both $C$ and $I^{*}$. So, we propose an empirical formula given by

$$
C_{D}=7 / 6 \text {. }
$$

Incidentally speaking, in Fig. 8, we also draw the experimental data by Dupleich ${ }^{(3)}$ which are quoted by Poreh \& Wrey ${ }^{(8)}$. We can see a qualitative discrepancy between the present and Dupleich where $C_{D}$ rapidly decreases with increasing $I^{*}$ at $I^{*}<3$. As one possible explanation for this inconsistency, we might suggest low values of $A R$ in his experiment. However, it is not easy to discuss precisely in the present stage, because of his insufficient descriptions of governing parameters and because of the lack of our enough knowledge on the $A R$ effects. Specifically speaking, concerning the former reason, we cannot know which of Dupleich's data Poreh \& Wray use. And thus, such governing parameters as $\lambda, A R$ and $C$ are possibly not constant, but vary within some ranges. Concern the latter reason, further investigations are required in future.

In Fig. 9, we first see the data for $C=7.2 \times 10^{1}$. According to both Figs. 7 and $8, C_{L} / C_{D}$ monotonously increases with increasing $I^{*}$, asymptoting to a constant value of about $6 / 5$, while there exist small data scatterings. Then, as well as Fig. 7, we can observe such similar effects of $I^{*}$ upon $C_{L} / C_{D}$ for $C=1.1 \times 10^{2}$ and $2.0 \times 10^{2}$ as that for $C=7.2 \times 10^{1}$. However, for $C \geq 3.7 \times 10^{2}, C_{L} / C_{D}$ monotonously decreases with increasing $I^{*}$, asymptoting to about $6 / 5$. From Eqs. (21) and (22), we get an empirical formula given by

$$
C_{L} / C_{D}=6 / 7 \alpha_{L}\left(1-\beta_{L} e^{-\gamma_{L} I^{*}}\right)
$$

with $\alpha_{L}=7 / 5, \beta_{L}=1800 C^{-1.4}-0.5$ and $\gamma_{L}=0.5$. Thin solid lines in Fig. 9 represent this empirical formula Eq. (23). We can confirm that the lines are in good agreement with the experimental data, as well.

\subsection{Influence of $\operatorname{Re}\left(V_{d}\right)$}

In the last subsection, we regard $C$ and $I^{*}$ as two kinematic governing parameters, and investigated the effects of $I^{*}$ upon such quantities as $\Omega^{*}, C_{L}, C_{D}$, and $C_{L} / C_{D}$ under the condition that $C$ keeps constant to one of six values. As a result, we have successfully proposed simple empirical formulae to predict those quantities with enough accuracies.

On the other hand, it is possible to choose other sets of two kinematic governing parameters. Now, we attempt another set, namely, a pair of $C$ and $\operatorname{Re}\left(V_{d}\right)$ or a pair of $I^{*}$ and $\operatorname{Re}\left(V_{d}\right)$, instead of a pair of $C$ and $I^{*}$.

As is mentioned before, the relation among $C, I^{*}$ and $\operatorname{Re}\left(V_{d}\right)$ is given by Eq. (9). Figure 10 shows the relation between $I^{*}$ and $\operatorname{Re}\left(V_{d}\right)$ for six constant values of $C$, on the $I^{*}-\operatorname{Re}\left(V_{d}\right)$ plane. That is, each dashed line in the figure represents Eq. (9) for one of six values of $C$. -Each solid line will be explained later--

Figure 10 also shows experimental values of $R e$ plotted against $I^{*}$ for the six $C$ 's, where the symbols as is described in Table 2. All the experimental Re's are always somewhat smaller than $\operatorname{Re}\left(V_{d}\right)$. This suggests that the actual value of $C_{D}$ is larger than unity which is assumed in Eq. (8).

Figure 11 shows $\operatorname{Re}$ plotted against $\operatorname{Re}\left(V_{d}\right)$, for $C=7.2 \times 10^{1}-1.6 \times 10^{3}$ and $I^{*}=0.75-$ 43. All the symbols in the figure correspond to those in Table 2, as well. In general, we should consider $\operatorname{Re}$ as a function of both $C$ and $\operatorname{Re}\left(V_{d}\right)$, or both $I^{*}$ and $\operatorname{Re}\left(V_{d}\right)$, in this context. However, we can suppose that the relation between $\operatorname{Re}$ and $\operatorname{Re}\left(V_{d}\right)$ is approximately determined as a function with uniqueness. Figure 11 proves this, although there exist small dispersions. In spite of wide variations of $C$ and $I^{*}$ in the experimental data, the relation can be given by

$$
\operatorname{Re}=0.78 \operatorname{Re}\left(V_{d}\right)
$$


with a good approximation.

Again, we see Fig. 10. Each solid line in the figure represents the relation Eq. (24) for one of six values of $C$. We can confirm that Eq. (24) shows a good approximation.

The fact that $\operatorname{Re}\left(V_{d}\right)$ is approximately a unique governing parameter of $R e$ implies such a possibility as $\operatorname{Re}\left(V_{d}\right)$ could be a unique governing parameter of aerodynamic-characteristics quantities such as $\Omega^{*}, C_{L}, C_{D}$ and $C_{L} / C_{D}$. As two examples, Figs. 12 and 13 show the $\operatorname{Re}\left(V_{d}\right)$ effects upon $\Omega^{*}$ and $C_{L} / C_{D}$, respectively. Again, Table 2 summarises the symbols and their corresponding parameters used in these figures. And, a solid line in each figure denotes the a proposed empirical formula, which will be discussed below.

In Figs. 12 and 13, $\Omega^{*}$ and $C_{L} / C_{D}$ increase with increasing $\operatorname{Re}\left(V_{d}\right)$, asymptoting to constant values, respectively. We should note that data scatterings are rather small, in spite of wide variations of $C$ and $I^{*}$. To conclude, $\Omega^{*}$ and $C_{L} / C_{D}$ are well approximated as follows.

$$
\begin{aligned}
& \Omega^{*}=4 / 7\left(1-e^{-0.0045 \operatorname{Re}\left(V_{d}\right)}\right) . \\
& C_{L} / C_{D}=6 / 5\left(1-e^{-0.0045 \operatorname{Re}\left(V_{d}\right)}\right) .
\end{aligned}
$$

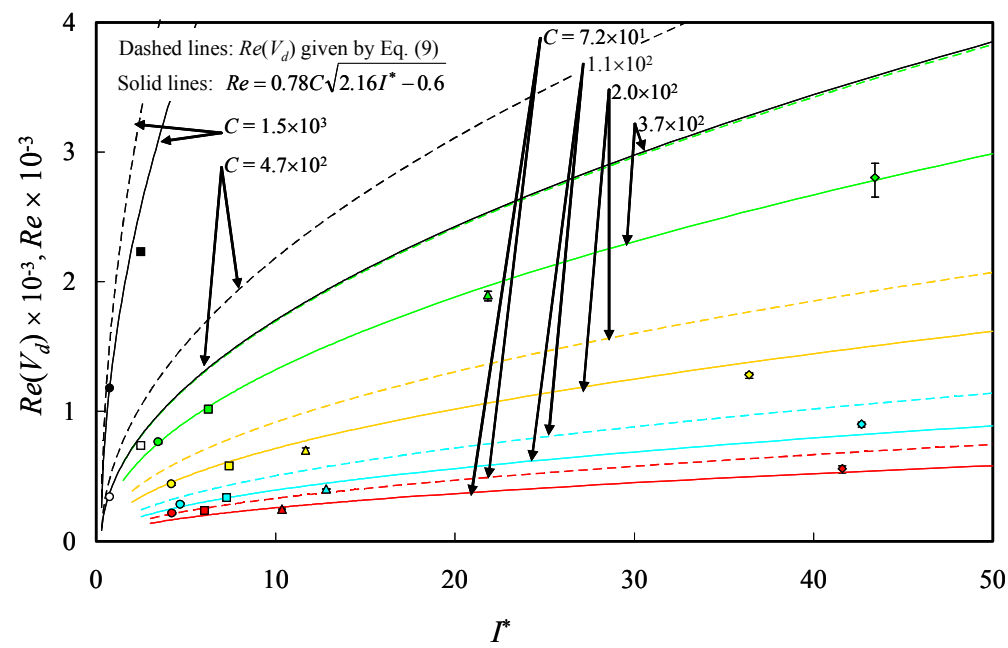

Fig. 10 Relation between $I^{*}$ and the Reynolds number $\operatorname{Re}\left(V_{d}\right)$ based on $V_{d}$ for six constant values of $C$, together with Reynolds number $R e$ versus inertia moment ratio $I^{*}$, for $\lambda=0.3, A R=10, C=7.2 \times 10^{1}-$ $1.6 \times 10^{3}$ and $I^{*}=0.75-43$.

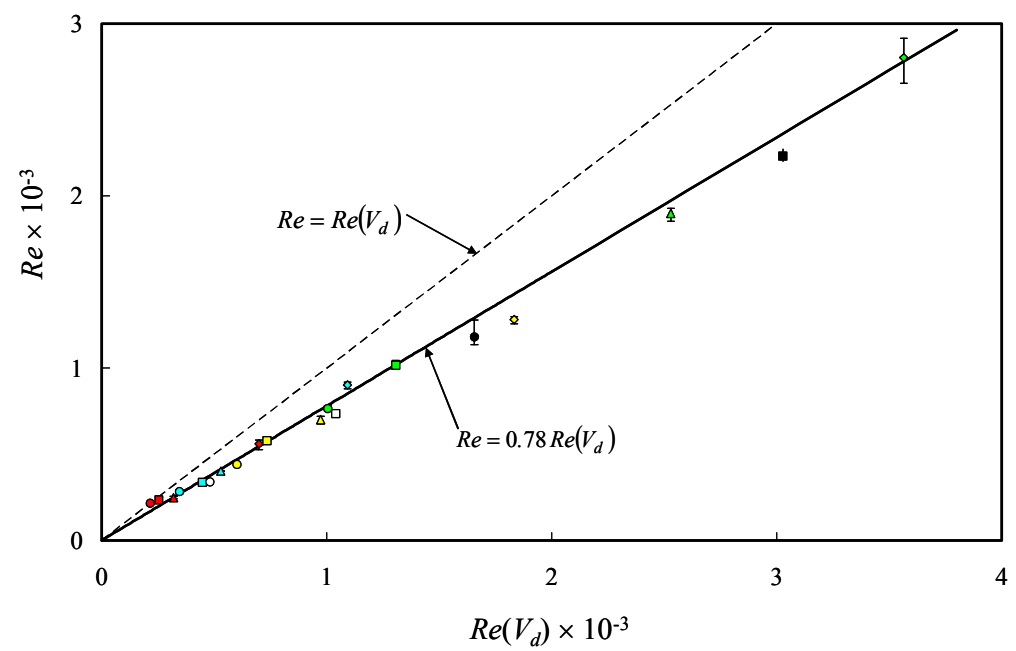

Fig. 11 Reynolds number $\operatorname{Re}$ versus the Reynolds number $\operatorname{Re}\left(V_{d}\right)$ based on $V_{d}$, for $\lambda=0.3, A R=10, C=$ $7.2 \times 10^{1}-1.6 \times 10^{3}$ and $I^{*}=0.75-43$. 
A solid line in each figure denotes these empirical formulae. And, according to Eq. (22), $C_{L}$ is given by

$$
C_{L}=7 / 5\left(1-e^{-0.0045 \operatorname{Re}\left(V_{d}\right)}\right),
$$

which is also in good agreement with the experimental data.

Of course, $\operatorname{Re}\left(V_{d}\right)$ might not always be a unique governing parameter, from a theoretical viewpoint. As an example, we consider the relation between $I^{*}$ and $C_{L} / C_{D}$ as shown in Fig. 9, again. Figure 14 shows $C_{L} / C_{D}$ plotted against $I^{*}$, for $C=7.2 \times 10^{1}-1.6 \times 10^{3}$ and $I^{*}=0.75$ -43 , whose experimental data are the same as those in Fig. 9. However, each solid line in the figure represents not the relation Eq. (23), but the relation Eq. (26) with the strict relation Eq. (9) among $C, I^{*}$ and $\operatorname{Re}\left(V_{d}\right)$, for one of six values of $C$. As a result, we can confirm less accuracy of Eq. (26) for large $C$ and small $I^{*}$.

Incidentally speaking, we can get the relation between $R e$ and $\Omega^{*}$ or that between $\operatorname{Re}\left(V_{d}\right)$ and $\Omega^{*}$, using Eq. (18) together with the relation Eq. (24) between $\operatorname{Re}\left(V_{d}\right)$ and $\operatorname{Re}$ in Fig. 11 and with the relation Eq. (9) among $C, I^{*}$ and $\operatorname{Re}\left(V_{d}\right)$. This also gives us another formula to predict $\Omega^{*}$ from two of $C, I^{*}$ and $\operatorname{Re}\left(V_{d}\right)$. In Fig. 15, this semi-theoretical formula is drawn by solid lines for four fixed values of $I^{*}$, and by dotted lines for four fixed values of $C$, together with the same empirical data in Fig. 5. For comparison, we also show the

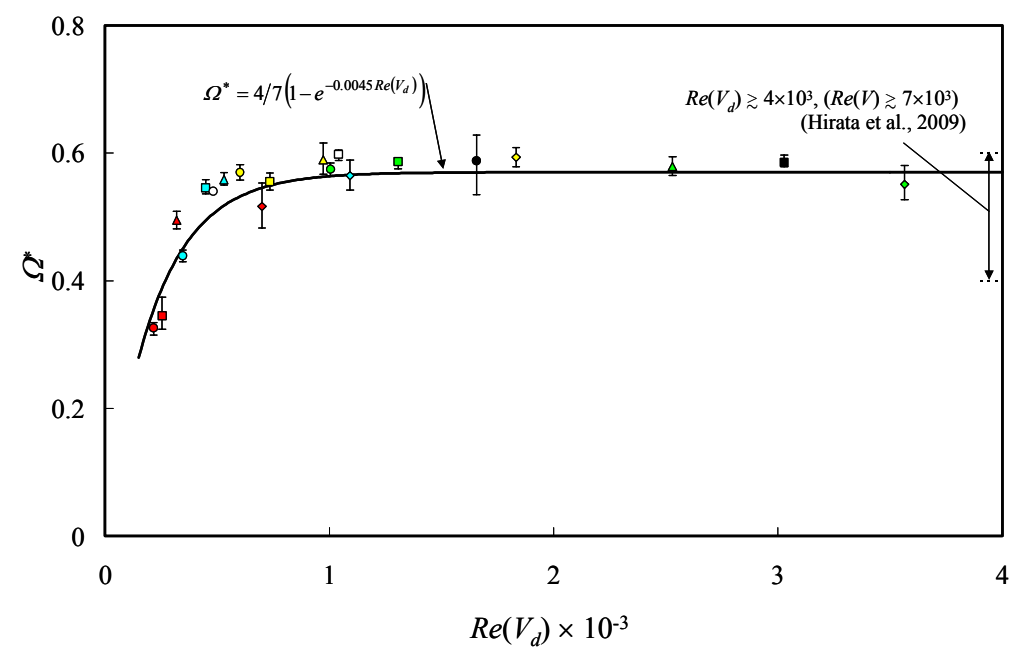

Fig. 12 Reduced rotating rate $\Omega^{*}$ versus the Reynolds number $\operatorname{Re}\left(V_{d}\right)$ based on $V_{d}$, for $\lambda=0.3, A R=10, C$ $=7.2 \times 10^{1}-1.6 \times 10^{3}$ and $I^{*}=0.75-43$.

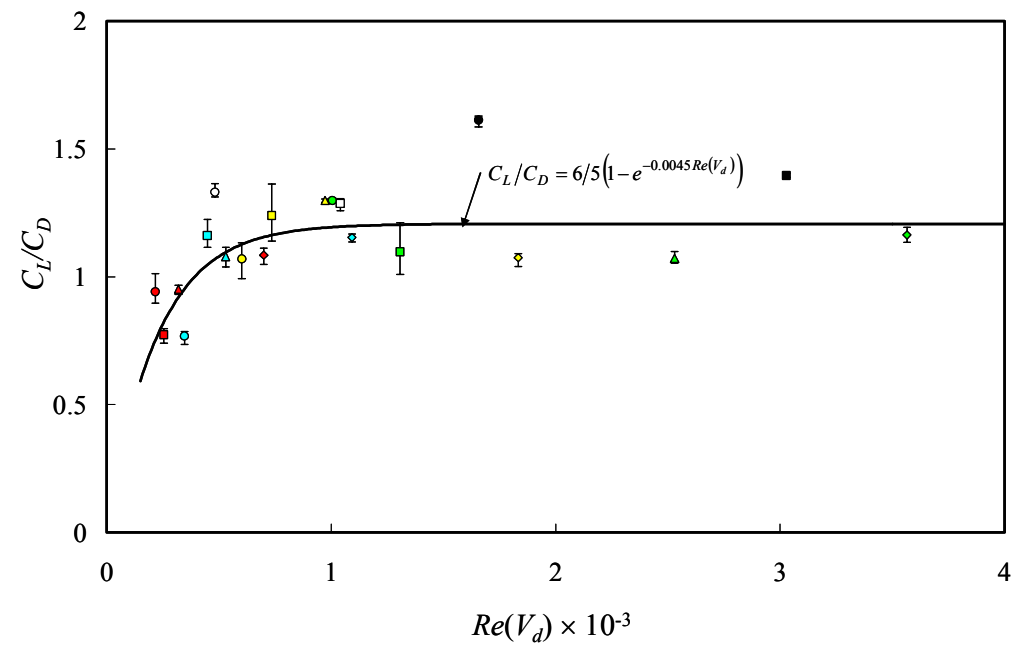

Fig. 13 Lift-drag ratio $C_{L} / C_{D}$ versus the Reynolds number $\operatorname{Re}\left(V_{d}\right)$ based on $V_{d}$, for $\lambda=0.3, A R=10, C=$ $7.2 \times 10^{1}-1.6 \times 10^{3}$ and $I^{*}=0.75-43$. 
empirical formula Eq. (25) with/without Eq. (24) which is drawn by a thick solid line. Unfortunately, the semi-theoretical formula based on Eq. (18) with Eqs. (24) and (9) seems less accurate than the empirical formula Eq. (25) with/without Eq. (24), and of course than Eq. (20).

\section{Conclusions}

We have experimentally dealt with the tumbling of a two-dimensional plate under free flight. Then, we have specified fundamental aerodynamic characteristics in terminal condition, like the reduced rotating rate $\Omega^{*}$, the lift coefficient $C_{L}$, the drag coefficient $C_{D}$ and the lift-to-drag ratio $C_{L} / C_{D}$. And, we have successfully proposed a series of empirical formulae to predict $\Omega^{*}, C_{L}, C_{D}$ and $C_{L} / C_{D}$ as functions of both a control parameter $C$ and the inertia moment ratio $I^{*}$, which can be available for wide governing-parameter ranges such as $C=7.2 \times 10^{1}-1.6 \times 10^{3}$ and $I^{*}=0.75-43$. Moreover, the relation between the actual Reynolds number $\operatorname{Re}$ and the Reynolds number $\operatorname{Re}\left(V_{d}\right)$ based on a theoretical velocity $V_{d}$ can be approximately determined, being independent of $C$ and $I^{*}$. Eventually, we have

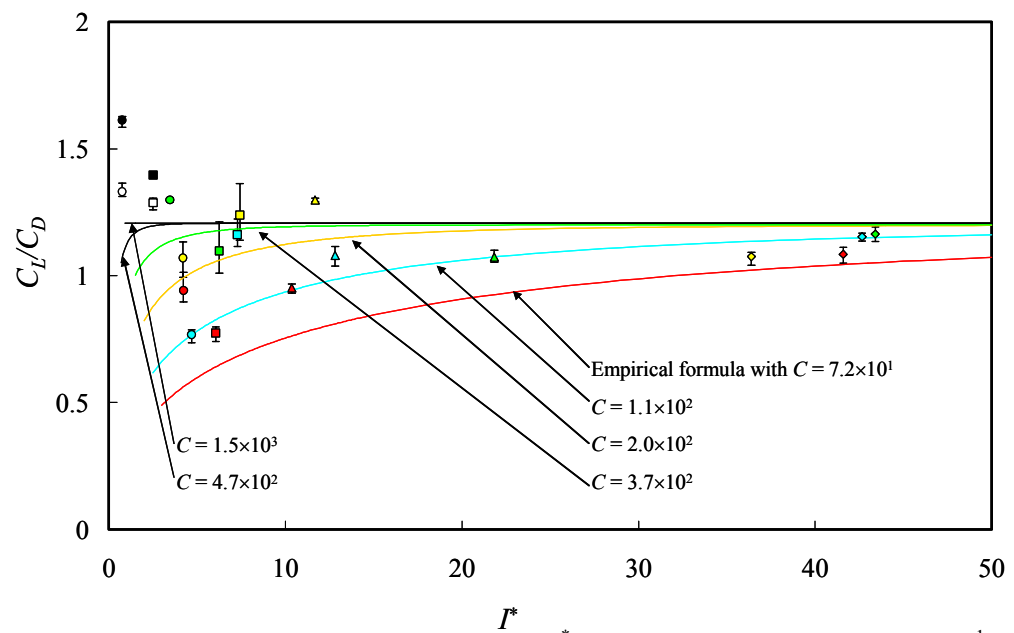

Fig. 14 Lift-drag ratio $C_{L} / C_{D}$ versus inertia moment ratio $I^{*}$, for $\lambda=0.3, A R=10, C=7.2 \times 10^{1}-1.6 \times 10^{3}$ and $I^{*}=0.75-43$. Empirical formula is given by Eq. (26).

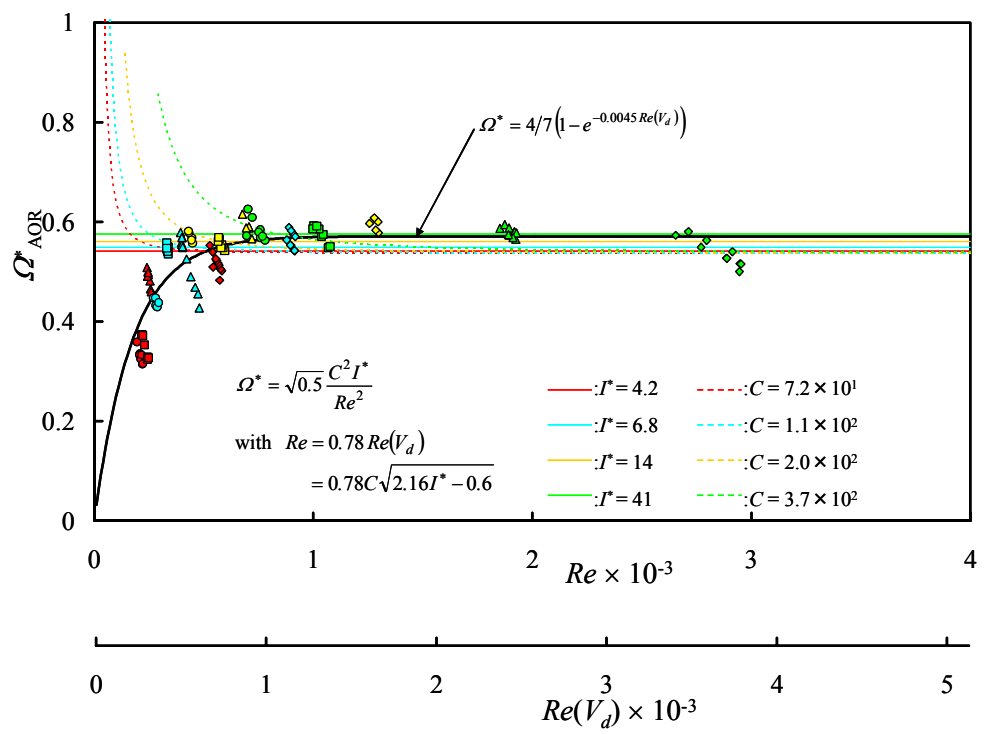

Fig. 15 Reduced rotating rate $\Omega_{\text {AOR }}^{*}$ averaged over one rotation versus Reynolds number $R e$, for $\lambda=0.3$, $A R=10, C=7.2 \times 10^{1}-3.7 \times 10^{2}$ and $I^{*}=4.2-43$. 
successfully proposed another series of empirical formulae to predict $\Omega^{*}, C_{L}, C_{D}$ and $C_{L} / C_{D}$ as functions of $\operatorname{Re}\left(V_{d}\right)$ alone, which is available for a wide parameter range except for large $C$ and small $I^{*}$. In addition, the scattering manner of $\Omega^{*}$ is well approximated by a semi-theoretical formula for in wide ranges of $C$ and $I^{*}$. This semi-theoretical formula is reflected by a close interaction based on the dynamical equilibrium on a free-flight plate supposing that the lift force $L$ is produced mainly by the Magnus effect. Unfortunately, the semi-theoretical formula is likely to be less accurate than the other two empirical formulae.

\section{References}

(1) H. J. Lugt, Autorotation, Annual Review of Fluid Mechanics, 15, 1983, pp. 123 - 147.

(2) T. Yoshinaga and A. Tate, Autorotation of slender bodies, Transactions of Japanese Society for Aeronautical and Space Sciences, 33, 1985, pp. 367 - 373 (in Japanese).

(3) P. Dupleich, Rotation in free fall of rectangular wings of elongated shape, NACA Technical Memorandum, 1201, 1949, pp. 1 - 99.

(4) A. M. O. Smith, On the motion of a tumbling body, Journal of The Aeronautical Sciences, 20, 1953, pp.73-84.

(5) A. C. Bustamante and G. W. Stone, The autorotation characteristics of various shapes for subsonic and hypersonic flows, AIAA Paper, No. $69-132$, 1969, pp. $1-11$.

(6) E. H. Smith, Autorotating wings, an experimental investigation, J. Fluid Mech., 50, 1971, pp. $513-534$.

(7) J. D. Iversen, Autorotating flat-plate wings, the effect of the moment of inertia, geometry and Reynolds number, J. Fluid Mech., 92, 1979, pp. 327 - 348.

(8) M. Poreh and R. N. Wray, On the motion of rectangular prismatic bodies, Journal of Fluids Engineering, 101, 1979, pp. 193 - 199.

(9) T. Yoshinaga, K. Inoue and A. Tate, Determination of the pitching characteristics of tumbling bodies by the free-rotation method, Journal of Spacecraft and Rockets, 21, 1984, pp. $21-28$.

(10) Y. Ishida, A Numerical Study of flow past a rotating flat plate by the discrete vortex method, Transactions of Japanese Society for Aeronautical and Space Sciences, 25, 1982, pp. 114 125.

(11) Y. Oshima, N. Izutsu, K. Oshima and K. Kuwahara, Autorotation of elliptic airfoil, AIAA Paper, No. 83 -0130, 1983, pp. $1-8$.

(12) H. J. Lugt, Autorotation of an elliptic cylinder about an axis perpendicular to the flow, $J$. Fluid Mech., 99, 1980, pp. $817-840$.

(13) A. Andersen, U. Pesavento and Z. J. Wang, Unsteady aerodynamics of fluttering and tumbling plates, J. Fluid Mech., 541, 2005, pp. 65 - 90.

(14) T. Yasuda and Y. Takano, Studies of flow pattern and fluid dynamic forces around an rotating elliptic cylinder (1st report, comparison between wind-tunnel experiment and numerical analysis based on finite volume method), Transactions of Japanese Society of Mechanical Engineers, Series B, 72, 2006, pp. 1404 - 1409 (in Japanese).

(15) K. Hirata, K. Shimizu, K. Fukuhara, K. Yamauchi, D. Kwaguchi and J. Funaki, Aerodynamic Characteristics of a Tumbling Plate under Free Flight, Journal of Fluid Science and Technology, 4, 2009, pp. $168-187$. 